\title{
AVALIAÇÃO DA FITODISPONIBILIDADE DE CHUMBO, CÁDMIO E CRÔMIO NO TABACO UTILIZADO NO NARGUILE
}

\author{
EVALUATION OF THE PHYTO-AVAILABILITY OF LEAD, CADMIUM AND \\ CHROMIUM IN TOBACCO USED IN NARGUILE
}

\author{
Daniel Ângelo Macena, Daniela Vanessa Bianchi, Hugo Favaretto \\ Menossi1, Vinícius Marques Gomes \\ Universidade do Oeste Paulista, Curso de Química Bacharelado, Presidente Prudente, \\ São Paulo. \\ e-mail: daniel@unoeste.br
}

RESUMO - Neste estudo foi abordado o uso da essência de Narguile que é utilizado para fumar tabaco aromatizado, muito popular nos países orientais. 0 presente estudo teve como objetivo quantificar metais contaminantes, como o Chumbo, Cádmio e Crômio, em amostras de essências utilizadas no consumo do Narguile. Como metodologia foi utilizada a técnica de Espectrometria de Absorção Atômica a mais utilizada para análises elementares metálicas em níveis de mg.L-1. Desta forma foi possível quantificar os metais e identificar a ordem de disponibilidade de cada metal, sendo $\mathrm{Cd}>\mathrm{Cr}>\mathrm{Pb}$. Representando numa média geral entre as cinco amostras 59\% do metal disponível foi o Cádmio, 37\% Crômio e por fim 4\% de Chumbo. Conclui-se que devido à variedade dos metais potencialmente tóxicos presentes e os efeitos colaterais que podem ser causados de acordo com a literatura, recomenda-se a minimização destes contaminantes através de autoridades e/ou possíveis órgãos regulamentadores.

Palavras-chave: tabaco aromatizado; metais tóxicos; essência de narguile; espectrometria de absorção atômica.

ABSTRACT - This study was approached the use of essence of Hookah that is used to smoke tobacco flavored, very popular in Eastern countries. The present study aimed to quantify contaminating metals, such as lead, cadmium and chromium, in samples of essences used in the consumption of narghile. As a methodology, it was used the Atomic Absorption Spectrometry technique the most used for elemental metal analysis at mg.L-1 levels. In this way, it was possible to quantify the metals and to identify the order of availability of each metal, with $\mathrm{Cd}>\mathrm{Cr}>\mathrm{Pb}$. Representing in a general average between the five samples $59 \%$ of the available metal was Cadmium, $37 \%$ Chromium and finally $4 \%$ Lead. It is concluded that due to the variety of potentially toxic metals present and the side effects that can be caused according to the literature, it is

Recebido em: 02/08/2017 Revisado em: 03/08/2017 Aprovado em: 07/08/2017 recommended to minimize these contaminants through authorities and/or public agencies.

Keywords: flavored tobacco; toxic metals; essence of hookah; atomic absorption spectrometry. 


\section{INTRODUÇÃO}

O Narguile, conhecido também como Cachimbo Árabe, Shisha, Hookah, Waterpipe, entre outros nomes; é uma espécie de cachimbo d'água e está dentre uma das formas não habituais utilizadas para fumar tabaco. Teve sua origem no oriente e é um hábito cultural de vários países; como a Índia, África e países do Oriente Médio (BROCKMAN et al., 2012).

O Narguile teve sua expansão quando exportado da Pérsia para a Arábia, onde os árabes incorporaram o cachimbo d'água para ser apreciado em grupos (FLOOR, 2002; AMERICAN LUNG ASSOCIATION, 2007; COBB,
2010). Foi introduzido no Brasil pelos descendentes árabes, sua maioria libaneses. O Narguile começou a tornar-se popular no fim dos anos 90 e desde então conta com adeptos em todo território nacional (NOVACZYK, 2010).

O Narguile é formado basicamente por uma base, corpo, fornilho e mangueira (FIGURA 2). A base ou vaso é o recipiente onde fica a água que resfria a fumaça e realiza a filtração, geralmente é feita de vidro, metal ou cerâmica (AMERICAN LUNG ASSOCIATION, 2007; NOVACZYK, 2010; MANSUR, 2013).

Figura 2. Estrutura e componentes básicos para o funcionamento do Narguile.

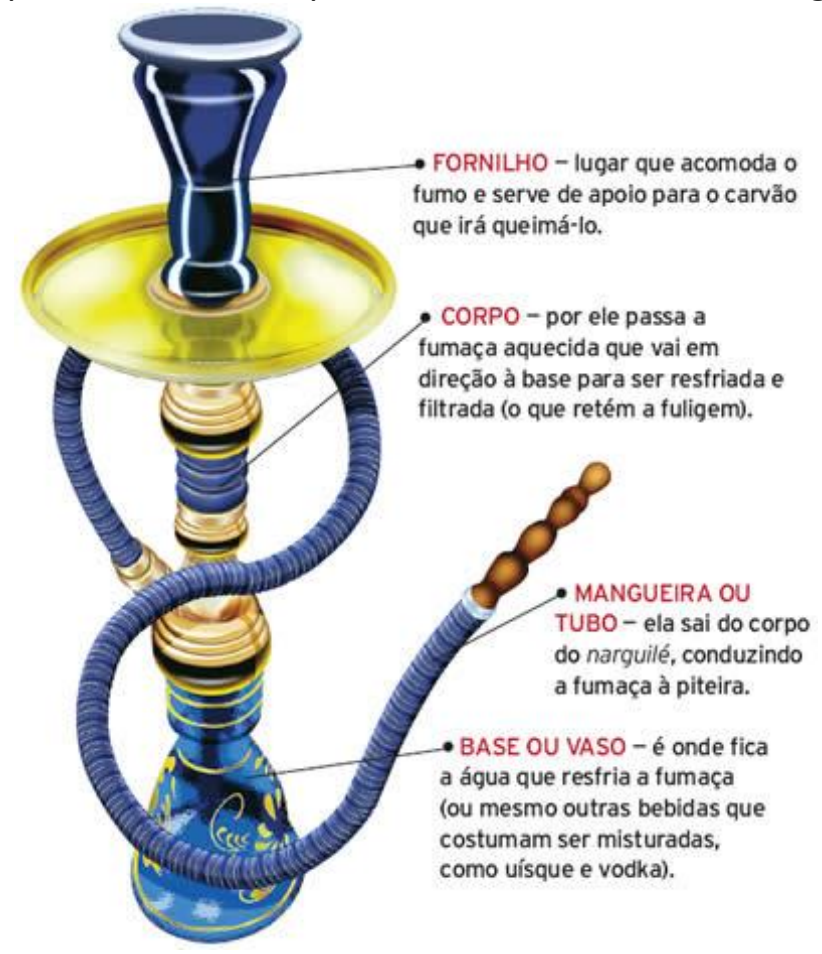

Fonte: Narguile Shon (2013)

A planta do tabaco, também utilizada na essência do Narguile, absorve do solo alguns elementos dos quais são considerados micronutrientes, tais como Cobre, Zinco, Manganês, Níquel, Ferro e Cobalto. No meio de transporte e absorção da planta pode 
ocorrer à absorção de elementos potencialmente tóxicos, provavelmente disponíveis de forma antrópica, como Arsênio, Chumbo, Cádmio e Crômio que torna um produto maléfico causando efeitos indesejados ao organismo (HAUSTEIN; GRONENERG, 2010 apud SILVA, 2015).

Os níveis de Chumbo, Cádmio e Crômio presentes na fumaça da combustão do tabaco são relativamente pequenos, porém com um potencial tóxico alto, devido à exposição crônica e suas propriedades redox-ativas (CARUSO et al., 2014).

Portanto este trabalho teve como objetivo determinar de forma quantitativa a presença de metais contaminantes, como o Chumbo, Cádmio e Crômio, em amostras representativas de essências de Narguile comercializadas legalmente no Brasil.

\section{METODOLOGIA}

\section{Aquisição das amostras}

Para a realização dos estudos foram selecionadas cinco marcas de essências de Narguile, todas de sabor mentolado, adquiridas em um estabelecimento de produtos para tabacaria, localizado no centro de Presidente Prudente. As embalagens de essências obtidas estavam devidamente lacradas, contendo 50 gramas cada um. Foram mantidas em temperatura ambiente até a realização das análises.

\section{Limpeza da vidraria}

Foram utilizados tubos digestores de Kjeldahl $(50 \mathrm{~mm} \times 250 \mathrm{~mm} \quad x \quad 2,5 \mathrm{~mm})$ higienizados em banho de ácido nítrico 10\% (v/v) por um período de 24 horas, lavados três vezes com água de osmose reversa e secos à temperatura ambiente (MESQUITA, 2014).

O banho em solução de ácido nítrico tem como finalidade a descontaminação de interferentes de metais residuais de análises anteriores. O metal impregnado na vidraria reage com a solução, formando nitratos, que por sua vez são solúveis em água.

\section{Tratamento das amostras}

Para a realização da digestão das amostras foi utilizado uma adaptação do método US - EPA 3050B (EPA, 1996). As extrações foram realizadas em duplicatas em bloco digestor para tubos macro de 8 provas a $120^{\circ} \mathrm{C}$. Para cada extração pesou-se aproximadamente $2,0 \mathrm{~g}$ de essência nos respectivos tubos, previamente higienizados e identificados, adicionando $20 \mathrm{~mL}$ de ácido nítrico (HNO3) concentrado.

Seguiu em aquecimento por 2 horas. A cada intervalo de 30 minutos foi adicionado $1 \mathrm{~mL}$ de peróxido de hidrogênio (H2O2) 30\% $\mathrm{v} / \mathrm{v}$, totalizando $4 \mathrm{~mL}$ no término de 2 horas.

$$
\text { Após resfriamento natural a }
$$
temperatura ambiente, os extratos obtidos foram filtrados com água de osmose reversa e transferidos para balões volumétricos de 
$100 \mathrm{~mL}$ e acertados até o menisco para aquisição do volume final.

Em seguida, os extratos diluídos foram transferidos para vidros âmbar estéreis onde ficaram armazenados e refrigerados à temperatura de $4^{\circ} \mathrm{C}$ até a realização das análises.

\section{Quantificação dos metais Chumbo, Cádmio e}

\section{Crômio}

Para a determinação dos teores de $\mathrm{Pb}, \mathrm{Cd}$ e $\mathrm{Cr}$, foi confeccionada uma curva analítica padrão de cada metal analisado para calibração do Espectrofotômetro de Absorção Atômica de chama (FAAS), da marca Perkin Elmer - Modelo AAnalyst 200 equipado com uma lâmpada de deutério como corretor de fundo e lâmpadas de cátodo-oco contendo cada analito, foi utilizado gás acetileno para atomização dos metais.

\section{RESULTADOS}

Na Tabela 1 segue os dados experimentais conforme a média de cada metal disponível, nas diferentes marcas e o desvio padrão para indicar os pontos de dados que tendem a estar próximos ao valor da média. É possível conhecer também a origem de cada essência, ao que foi levado em consideração 5 marcas diferentes consumidas no mercado brasileiro, por tanto não houve relevância significativa quanto as origens serem iguais. $\mathrm{O}$ peso médio obtido das amostras foi de 2,0433 $\mathrm{g} \pm 0,0368$.

Tabela 1. Dados experimentais da concentração de metal disponível (Cádmio, Crômio e Chumbo) em cada marca e sua origem.

\begin{tabular}{c|c|c|c|c}
\hline \multirow{2}{*}{ Marca } & \multicolumn{4}{|c}{ Concentração $\mu$ g.g $^{-1} \mathrm{MDE}$} \\
\cline { 2 - 5 } & Origem & Cd & $\mathrm{Cr}$ & $\mathrm{Pb}$ \\
\hline A & Jordânia & $12,60 \pm 1,10$ & $3,70 \pm 1,40$ & $1,00 \pm 0$ \\
\hline B & Turquia & $9,45 \pm 0,25$ & $10,60 \pm 4,80$ & $1,00 \pm 0$ \\
\hline C & Jordânia & $13,45 \pm 0,45$ & $2,70 \pm 1,00$ & $1,00 \pm 0$ \\
\hline D & Jordânia & $17,05 \pm 0,85$ & $3,70 \pm 1,60$ & $1,00 \pm 0$ \\
\hline
\end{tabular}

Fonte: Próprios autores (2017).

Nota: $\mathrm{MDE}=$ Metal Disponível na Essência.

Segue histograma (Gráfico 1) denominadas de A a E no estudo, para representando as 5 marcas diferentes preservar a imagem dos fabricantes, onde foi 
observado a disponibilidade de cada metal e

diferentes marcas.

a relação entre as quantidades e as

Gráfico 1. Dados da concentração de metais disponíveis em diferentes marcas de essência de Narguile com linhas verticais representando desvio padrão da média.

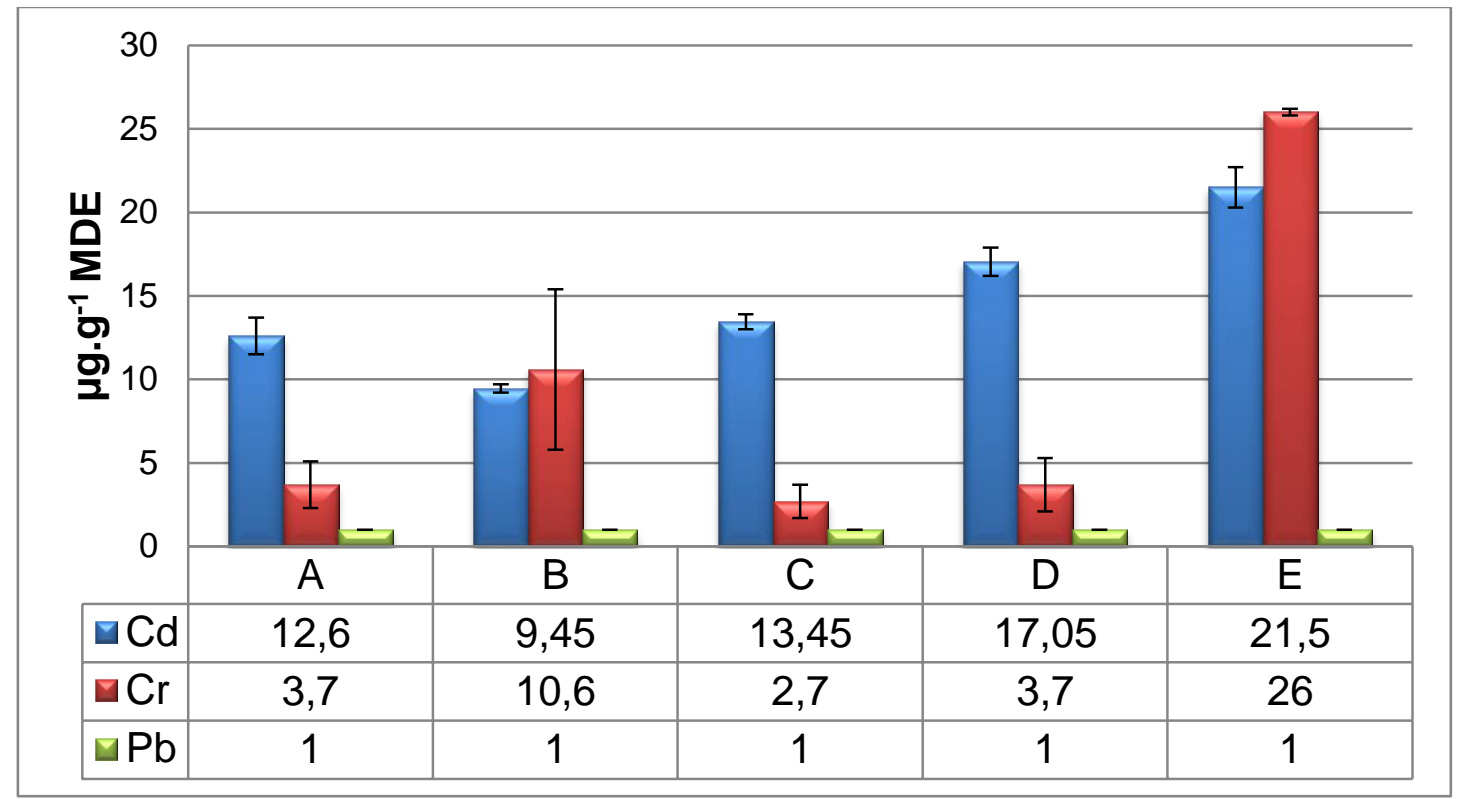

Fonte: Próprios autores (2017).

Nota: $\mathrm{MDE}=$ Metal Disponível na Essência.

O Gráfico 2 foi plotado a partir da média geral do metal Cádmio, Crômio e
Chumbo entre todas as marcas para análise geral e ordem de disponibilidade.

Gráfico 2. Média geral de metal Cádmio, Crômio e Chumbo disponíveis nas marcas A, B, C, D e E.

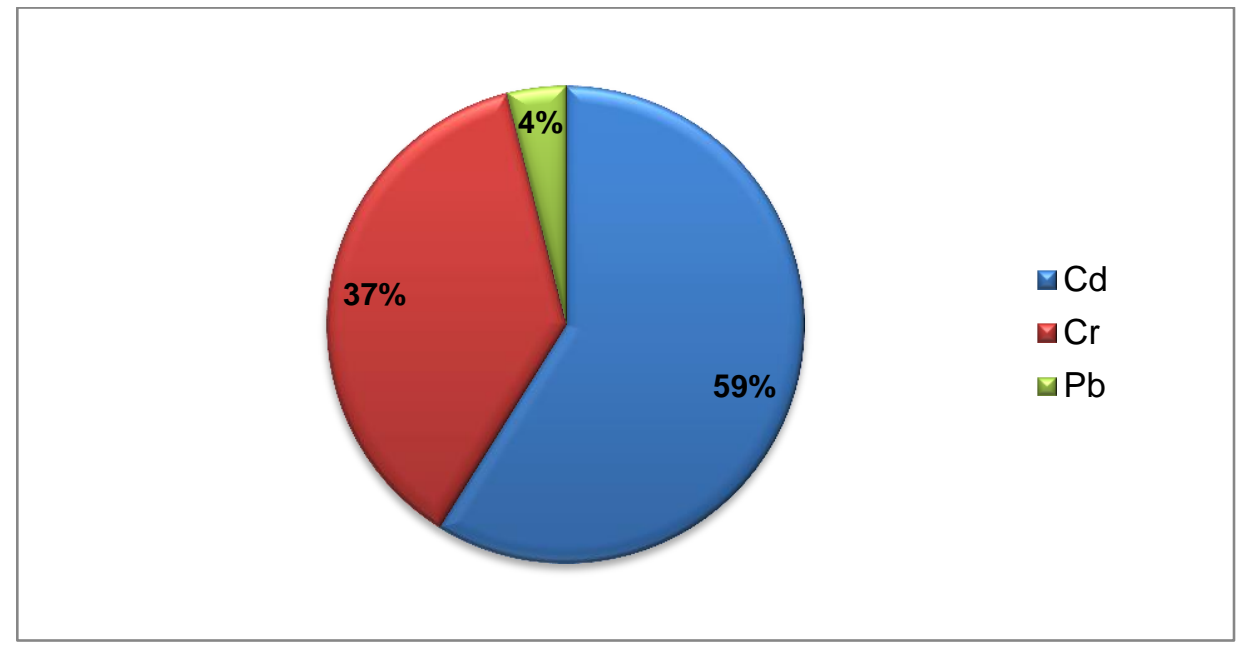

Fonte: Próprios autores (2017). 
O Gráfico 3 foi relacionado ao Gráfico 2, no qual foi feita uma observação entre a linha de máximos e mínimos a partir da média geral para comprovação da ordem de disponibilidade real de cada metal nas 5 amostras.

Gráfico 3. Linhas de máximos e mínimos apresentado nos resultados entre as diferentes marcas para cada metal analisado.

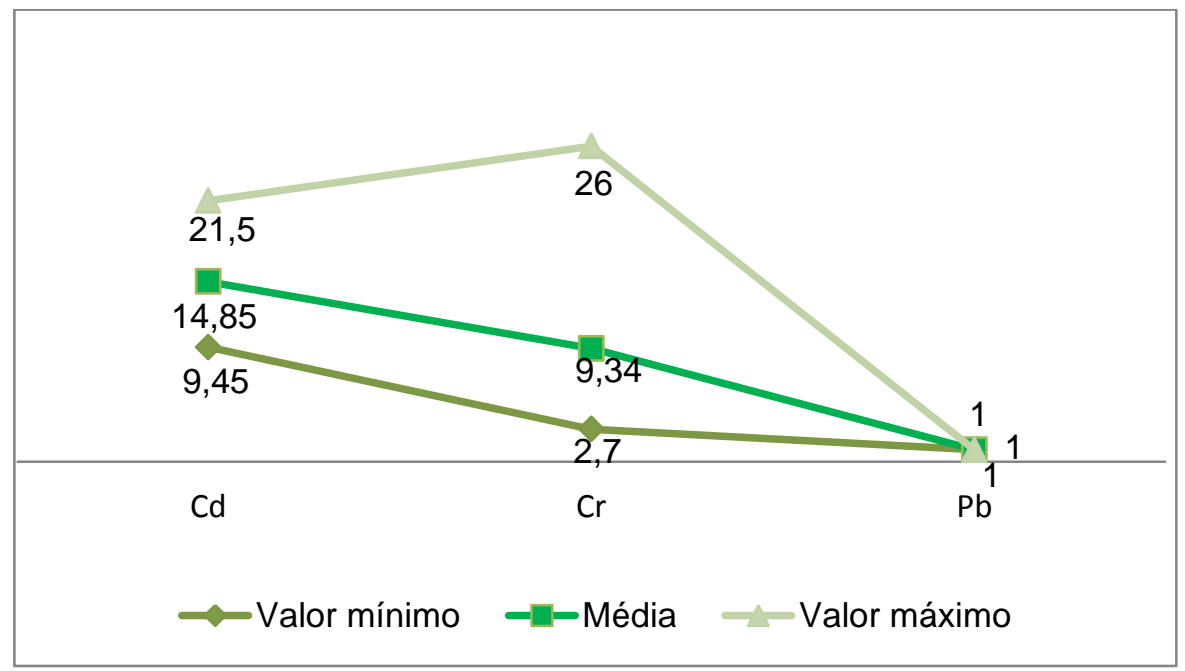

Fonte: Próprios autores (2017).

\section{DISCUSSÃO}

$\mathrm{Na}$ tabela 1 foi observado que a marca B foi o ponto que apresentou o maior desvio em uma gama de valores para o metal Crômio. Observou-se também que todas as amostras para Chumbo não houve variação, mostrando então que todas as replicatas obtiveram a mesma disponibilidade.

No Gráfico 1 observou-se maior disponibilidade de metais Cádmio e Crômio nas amostras sendo a menor concentração de Cádmio na amostra $B$ e a maior concentração a amostra E, mostrando-se mais de 2 vezes maior que a amostra B. Já para o Crômio, a maior concentração analisada foi a amostra $E$, sendo observado uma quantificação aproximadamente 9 vezes maior que a amostra C. Ainda sobre o Crômio, percebeu-se também uma semelhança na disponibilidade entre a marca A e $D$ na qual as concentrações foram iguais.

A média das maiores concentrações de Cádmio e Crômio foram determinadas na amostra de essência do país de origem Paraguai (marca E), enquanto a menor média destes metais foi analisada na essência de origem Jordânia. A diferença de disponibilidade pode estar também atrelada ao fato de as amostras serem de diferentes origens.

Pode-se observar no gráfico 2 que independentemente das diferentes concentrações disponíveis, o metal Cádmio é o que mais representa de acordo com a 
somatória entre as 5 marcas, correspondendo por uma fatia de 59\%, enquanto o Crômio 37\%, onde a ordem de disponibilidade nesta faixa amostral ficou Cádmio > Crômio > Chumbo.

Relacionando o gráfico 2 ao gráfico 3 percebe-se que mesmo a linha de máximos para Crômio ter sido mais alto, na média geral de disponibilidade o Cádmio apresenta

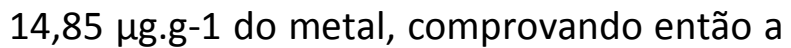
maior disponibilidade entre as marcas. Podese concluir ainda que, entre as linhas de máximos e mínimos está a média que demonstra nitidamente a ordem de disponibilidade de cada metal na média geral entre as 5 amostras de marcas diferentes de essência de Narguile.

De acordo com os estudos de AlKazwini, Said e Sdepanian (2015), na fumaça do Narguile após a queima da essência existem a presença de metais contaminantes, confirmando a teoria que os metais contidos na essência antes da queima são volatilizados através do calor e transportados pela fumaça até a inalação. Neste mesmo estudo, foram utilizadas marcas importadas da Jordânia e dos Emirados Árabes Unidos e os resultados apresentados pelo autor mostram que a teoria que se fundamentou durante o tempo, em que a água presente no vaso do Narguile funcionaria como um filtro de partículas e da fumaça não é verdade, pois a água retém apenas $3 \%$ dos metais volatilizados. Dentre os vários metais analisados foram quantificados também o Chumbo e o Crômio, tanto na essência quanto na fumaça ao qual apenas $22 \%$ de todo o Crômio presente na amostra se encontra após a queima da essência do Narguile, com isso os $78 \%$ restantes foram quantificados na fumaça. Enquanto que o Chumbo apresentou 63\% de todo o metal na fumaça.

Outro estudo por Pappas et al. (2015) foi determinado níveis de metais contaminantes em tabaco de cigarros, obtendo uma média de 20,04 $\mu$ g.g-1 para Pb;

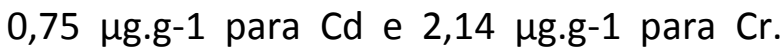
Comparando com os resultados do presente estudo pode-se perceber a grande diferença entre a disponibilidade de $\mathrm{Pb}$ sendo aproximadamente 20 vezes maior no cigarro do que na essência de Narguile, enquanto que os metais $\mathrm{Cd}$ e $\mathrm{Cr}$ ocorre o inverso em ordem de disponibilidade.

\section{CONCLUSÃO}

A técnica de Espectrometria de Absorção Atômica é a mais utilizada para análises elementares metálicas em soluções líquidas, sólidas ou gasosas em níveis de mg.L-1. No presente trabalho a técnica apresentou de forma eficiente na quantificação dos metais em estudo, tais como; $\mathrm{Cd}$, $\mathrm{Pb}$ e $\mathrm{Cr}$.

Conclui-se que independentemente da ordem de disponibilidade dos metais presente neste estudo, foi possível a 
detecção dos mesmos. Com isso, devido à variedade dos metais potencialmente tóxicos presentes e os efeitos colaterais que podem ser causados de acordo com a literatura, recomenda-se maior atenção quanto a fiscalização dos produtos disponíveis que adentram no Brasil afim da minimização destes contaminantes através de autoridades e/ou possíveis órgãos regulamentadores.

\section{REFERÊNCIAS}

AL-KAZWINI, A. T.; SAID, A. J.; SDEPANIAN, S. Compartmental analysis of metals in waterpipe smoking technique. BMC Public Health, v. 15, n. 1, p. 153, 2015. https://doi.org/10.1186/s12889-015-1373-6

AMERICAN LUNG ASSOCIATION. An emerging deadly trend: Waterpipe tobacco use. PDF222 KB). Washington: American Lung Association, 2007.

BROCKMAN, L. N. et al. Hookah's new popularity among US college students: a pilot study of the characteristics of hookah smokers and their Facebook displays. BMJ Open, v.2, n.6, 2012. https://doi.org/10.1136/bmjopen-2012$\underline{001709}$

CARUSO, R. V. et al. Toxic metal concentrationsin cigarettes obtained from U.S.Smokers in 2009: results from The International Tobacco Control(ITC) United States Survey Cohort. International Journal of Environmental Research and PublicHealth, v. 11, n. 1, p. 202-217, 2014.

COBB, C. et al. Waterpipe tobacco smoking: an emerging health crisis in the United States. American Journal Of Health Behavior, v. 34, n. 3, p. 275-285, 2010. Disponível em: <https://www.ncbi.nlm.nih.gov/pubmed/200 01185>. Acesso em: 27 jul. 2017.
FLOOR, W. The art of smoking in Iran and other uses of tobacco. Iranian Studies, v. 35, n.1-3, p.47-85, 2002. https://doi.org/10.1080/0021086020870201 $\underline{1}$

MANSUR, L. A Origem e História do Arguile. 2013. Disponível em: <http://www.gazetadebeirute.com/2013/07/ a-origem-e-historia-do-arguile.html>. Acesso em 18 nov. 2016.

MESQUITA, G. M. Metodologias de preparo de amostras e quantificação de metais pesados em sedimentos do Ribeirão Samambaia, Catalão-GO, empregando Espectrometria de Absorção Atômica. 2014. 134 f. Dissertação (Mestrado) - Universidade Federal de Goiás, Catalão, 2014.

NARGUILE SHON. Partes do Narguilé. 2013. Disponível

em: http://narguile2013.blogspot.com.br/2013/0 3/partes-do-narguile_15.html. Acesso em: 15 jul. 2017.

NOVACZYK, K. O que é a Shisha ou o Narguile?. 2010. Disponível em: <http://www.khanelkhalili.com.br/arguile.ht $\mathrm{m}>$. Acesso em 27 jul. 2017.

PAPPAS, R. S. et al. Determination of toxic metals in little cigar tobacco with triple quad'ICP-MS. Journal Of Analytical Toxicology, v. 39, n. 5, p. 347-352, 2015. https://doi.org/10.1093/jat/bkv016

SILVA, C. P. Caracterização e Avaliação da Qualidade dos Cigarros Contrabandeados no Brasil. 2015. 123 f. Dissertação (Mestrado em Química Aplicada) - Universidade Estadual de Ponta Grossa, Ponta Grossa-PR, 2015. 